\title{
Trial of long-term anticoagulant therapy in the treatment of small stroke associated with a normal carotid arteriogram
}

\author{
PETER BRADSHAW ${ }^{1}$ AND STEPHEN BRENNAN \\ From the Department of Neurology, United Sheffield Hospitals, Sheffield
}

SYNOPSIS The clinical features of 49 patients who had sustained small strokes in the internal $\overrightarrow{0}$

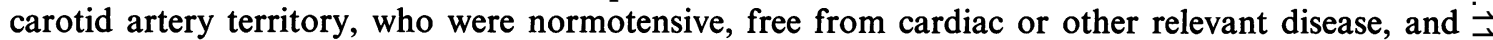
who each had a normal appropriate single vessel angiogram are presented. These were randomized into two groups: group A, 25 patients, who received only supportive treatment; group B, 24 patients, who were treated with anticoagulants for an average period of 18 months. There was a reduced incidence of neurological episodes during the administration of anticoagulant therapy but, after $\infty$ treatment was discontinued, there was no significant difference between the two groups. In view of $-v$ the relatively benign prognosis for this syndrome, unless special facilities exist for the personal $\frac{1}{\mathrm{~N}}$ control of anticoagulant treatment, the dangers may outweigh the benefits.

In a previous study of small strokes in the territory of the internal carotid artery, Bradshaw and Gumpert (1972) reported that appropriate single vessel angiography was normal in 50 of 69 consecutive patients. Thirty-five of the 50 who had normal arteriograms were normotensive and, when followed up for between six months and six years, it was found that three patients had died of related disease, three had been disabled by further stroke, and 29 were normal. Further, it was noted that all nine patients who had received long-term anticoagulant treatment had remained well. However, the benign nature of the syndrome and the paucity of patients treated with anticoagulants made evaluation of such treatment impossible. This paper is an extension of the previous study and presents a series of patients who had sustained small strokes in the internal carotid territory, were normotensive, without evidence of any cardiac source of emboli, and who had normal appropriate single vessel angiography. Twenty-four of these were treated with anticoagulant therapy and 25 received only supportive treatment.

1 Present address: The Royal Hospital Annexe, Fulwood, Sheffield S10 3TD.

(Accepted 21 February 1975.)
Hill et al. $(1960,1962)$ reported that the proĝ의 nosis of patients presenting with transierit $\overrightarrow{0}$ ischaemic attacks (TIAs) was not improved bै long-term treatment with anticoagulants. They 0 noted that their patients displayed an above average risk of intracranial haemorrhage and concluded that, in the absence of any therapeutic benefit, the dangers attendant upon use of $\mathscr{Q}$ anticoagulants made their use unacceptable. $\overrightarrow{\vec{D}}$ However, Millikan et al. (1955), Fisher (1958) 3 McDevitt et al. (1958), Siekert et al. (1961), Millikan (1971), and others, have all noted improvement in the prognosis of TIAs when anticoagulant therapy is employed. This conflict of opinion is in large measure due to lack of precise $\frac{5}{3}$ information concerning patients comprising the different series. In many of these publications there is no distinction made between TIAs in the internal carotid and vertebrobasilar circulations $?$ and, as Bradshaw and McQuaid (1963) have음 shown, the syndrome of vertebrobasilar insufficiency is associated with an especially benign of prognosis. Further, not all reported series indi- $N$ cate whether TIAs in the internal carotid artery $\tilde{\omega}^{N}$ territory were associated with occlusive carotido artery disease or occurred in patients witho normal carotid arteriograms. Acheson and 
Hutchinson (1964), Marshall (1964, 1968), and Bradshaw and Casey (1967) have all emphasized the grave prognosis of stroke associated with occlusive carotid artery disease, in which between 50 and $70 \%$ of patients either succumb or become severely disabled from further stroke; the majority within a few months of the onset of relevant symptoms.

It is also probable that other factors profoundly influence the prognosis of small strokes in the carotid artery territory. Leishman (1959, 1963) had indicated the grave outlook for patients with hypertension who remain untreated and many of these died or were disabled by stroke. Bradshaw and Gumpert (1972), in a review of small strokes with normal carotid arteriograms, found that nearly $50 \%$ of patients with hypertension fared badly, even though every effort had been made to control excessive blood pressure. Marshall and Wilkinson (1971) also suggested that the presence of heart disease might have an adverse effect on prognosis of small strokes. It would seem, therefore, that before any useful evaluation of any treatment for stroke can be made it is necessary to restrict the selection of treated and control groups to take into account the presence or absence of occlusive arterial disease, the state of the blood pressure, the presence or absence of heart disease, and the clinical pattern of stroke.

\section{METHODS}

SELECTION OF PATIENTS AND CLINICAL METHODS A prospective study was commenced on 1 January 1970 of patients who presented with small strokes in the territory of the internal carotid artery, who had neither hypertension nor significant heart disease, and in whom an appropriate percutaneous carotid arteriogram was normal. By a 'small stroke' is here understood either a TIA, or a small incomplete sudden stroke in which residual neurological signs persisted for more than 24 hours but which did not amount to more than very slight disability. The reason why patients with minimal residual signs were included is that when such are detected after two or more neurological episodes, it is often impossible to determine whether they arise in connection with the first or with subsequent attacks. Exclusion of such patients would bias the series in favour of a benign prognosis. All patients were admitted to the department of neurology of the United Sheffield Hospitals, at Fulwood, under personal care. Six hourly blood pressures were recorded for the first seven days and a label of normotensive was applied if this failed to show a diastolic blood pressure above $104 \mathrm{mmHg}$. Patients were excluded from the series for one or more of the following reasons: above the age of 65 years, diabetes mellitus, myxoedema, clinical or electrocardiographic evidence of relevant heart disease, clinical evidence of moderate to severe atheroma of limb vessels. Care was also exercised to avoid including patients who suffered from hemiplegic migraine. A total of 35 patients were accepted and divided into 18 in group $B$, to receive treatment with anticoagulants, and 17 patients in group $A$, to receive only supportive treatment. Randomization was effected by an odd or even year of birth.

The numbers in the two groups were increased by adding 14 other similar patients. These comprised the total number admitted to the department between March 1965 and December 1969 who fulfilled the necessary criteria. Six of these patients had received anticoagulant therapy and eight had received only supportive treatment on a random basis of selection, though randomization had not been effected in the same way as in the prospective study. Thus group A contained 25 and group B 24 patients.

Routine investigation included: full blood count and erythrocyte sedimentation rate (ESR); urinalysis: plain radiographs of chest and skull; serum electrolytes and blood urea; and a blood Wassermann reaction (WR). One or more electrocardiograms (ECG) were recorded. All but four patients had a lumbar puncture, and in one of these the lumbar cerebrospinal fluid (CSF) had been reported as normal elsewhere; in the three others the interval between presentation and initial assessment was more than three weeks. All patients had an appropriate percutaneous carotid arteriogram.

Patients in group A included 22 treated with warfarin, and two who received phenindione. Stabilization was effected in hospital and maintained with prothrombin times of approximately twice that of fresh controls throughout. After discharge, these patients were followed up at weekly or monthly intervals, as seemed appropriate, when the dosage of drugs was adjusted. Treatment was maintained for nine to 42 months in 23 patients and in one initially for only three months. Cessation of therapy was effected by tapering off the dosage over a period of four to six weeks, to avoid rebound phenomena as described by Marshall (1963). Patients in group B were followed up at intervals of two to six months.

Thirteen patients defaulted from follow-up appointments. Four of these had died-three in hospital elsewhere, one at home-and in all the cause of death was verified. Three others had kept attendances for not less than three years when they 
had been well, and up-to-date information was obtained by post from both patient and family doctor. Six were visited in their homes.

\section{RESULTS}

The average age of the 49 patients was 52 years; in the treated group A it was 53 (32-62) years, and in the untreated group B it was $51(30-64)$

TABLE 1

INTERVAL BETWEEN FIRST NEUROLOGICAL EPISODE AND FIRST ASSESSMENT

\begin{tabular}{cccc}
\hline Interval & Group A & Group $B$ & Total \\
\hline Days & 6 & 6 & 12 \\
$<7$ & 14 & 15 & 29 \\
$8-28$ & 4 & 4 & 8 \\
$\begin{array}{c}\text { Months } \\
3-12\end{array}$ & 24 & 25 & 49 \\
\hline
\end{tabular}

TABLE 2

NUMBER OF NEUROLOGICAL EPISODES BEFORE FIRST ASSESSMENT

\begin{tabular}{lrcc}
\hline Episodes & Group A & Group 3 & Total \\
\hline Single & 15 & 15 & 30 \\
$2-3$ & 6 & 7 & 13 \\
$4-5$ & 2 & -3 & 2 \\
Multiple & 1 & 3 & 49 \\
\hline
\end{tabular}

years. The ratio of men to women was 19:5 and 19:6 respectively. Two patients in each group gave a history of myocardial infarction more than 18 months before their first neurological episode. The time interval between the first relevant symptoms and initial assessment is shown in Table 1. It will be seen that, in 41 patients, admission to hospital was effected within 28 days of the first neurological episode and in eight others this was delayed for three to 12 months. The individual frequency of the episodes is shown in Table 2. Some 43 patients had between one and three attacks before they were first seen and
TABLE 3

DURATION OF NEUROLOGICAL EPISODES

\begin{tabular}{cccc}
\hline Duration $(h)$ & Group $A$ & Group B & Total \\
\hline$<1$ & 9 & 11 & 20 \\
$1-3$ & 3 & 3 & 6 \\
$4-24$ & 4 & 2 & 6 \\
$>24$ & 8 & 9 & 17 \\
\hline
\end{tabular}

TABLE 4

SYMPTOMS

\begin{tabular}{|c|c|c|c|}
\hline Symptoms & Group $A$ & Group B & Total \\
\hline Speech disturbance & 6 & 7 & 13 \\
\hline Monocular blindness & 2 & 2 & 4 \\
\hline Homonymous field defect & $\mathbf{1}$ & 2 & 3 \\
\hline Weakness and/or paraesthesiae & 21 & $2 \overline{3}$ & 44 \\
\hline
\end{tabular}

the duration of the first or only episode is shown in Table 3. In 32 patients the episodes lasted for less than 24 hours and might justifiably designated TIAs.

Table 4 shows the nature and distribution of neurological symptoms. It will be seen that 13 patients complained of speech disturbance which was in all thought to have been dysphasia. In three patients this was an isolated symptom, in $\cong$ 10 it was associated with other symptoms which $\overrightarrow{\overrightarrow{0}}$ in nine patients indicated involvement of the dominant and in one involvement of the subdominant hemisphere. Seven other patients complained of visual disorder. In four cases this amounted to monocular blindness and in two : of these it was the only symptom. In three 3 patients, it seemed to have been a homonymous defect of vision which in all instances was associated with other ipsilateral disorder to $ᄋ$ suggest that the carotid rather than the vertebro- $D$ basilar circulation was involved. Distinction between paraesthesiae and weakness seemed N often to be unclear in the patient's mind and no attempt has been made to distinguish between 0 them. In all, 44 patients had either weakness, $\omega$ paraesthesiae, or both which were referred almost invariably to the upper limb, less often to the lower limb, and occasionally to the face. The 
territory of the right internal carotid circulation was involved in 30 and the left in 19 cases.

Table 5 shows the nature and distribution of the neurological signs. Twenty-seven patients were neurologically normal. A bruit was heard over the appropriate side of the neck in three patients. Four patients had a mild expressive dysphasia, and in three of these there were no other abnormal signs. One patient displayed an isolated homonymous hemianopia, but this had been associated with transient dysphasia and weakness of the right upper limb suggesting that

TABLE 5

NATURE AND DISTRIBUTION OF PHYSICAL SIGNS

\begin{tabular}{lccc}
\hline Physical signs & Group A & Group B & Total \\
\hline Neck bruit & 1 & 2 & 3 \\
Homonymous hemianopia & - & 1 & 1 \\
Pyramidal tract signs & & & \\
$\quad$ Total & 10 & 10 & 20 \\
$\quad$ Upper limb & 9 & 8 & 17 \\
Lower limb & 4 & 3 & 7 \\
Face & 1 & 2 & 3 \\
Hemisensory change & 1 & 1 & 2 \\
Dysphasia & 1 & 3 & 4 \\
None & 14 & 13 & 27 \\
\hline
\end{tabular}

the circulatory disturbance involved the internal carotid rather than the vertebrobasilar arterial territory. Evidence of pyramidal tract involvement in 20 cases was found, and this consisted of no more than a catch of spasticity in the forearm, reduction of fine finger movement, relatively slight weakness, and an increase of deep tendon reflexes. In seven patients in whom the lower limb was also involved, the plantar response was extensor. There was also slight facial weakness in three cases. Two patients initially had apparent hemisensory change which resolved during the period in hospital. At the time of discharge from hospital, only five patients in group A and seven in group B had residual signs.

INVESTIGATIONS A full blood count and ESR were carried out in 49 patients and were normal in 47 ; in one there was an iron deficiency anaemia with an haemoglobin of $8.6 \mathrm{~g} / \mathrm{dl}$, which was corrected with oral iron in two months; in one other patient the ESR was $35 \mathrm{~mm}$ per hour, this was attributed to an $E$. coli urinary tract infection which responded to treatment with cotrimoxazole. Urinalysis was normal in 48 patients and showed the infection already mentioned in one. Plain radiographs of chest and skull were normal in 49 patients. An ECG was normal in 44 patients; in three there were changes of an old myocardial infarction, corresponding with a clinical history of such more than 18 months previously; another two patients had left axis deviation in their ECG. Blood urea and serum electrolytes were normal in 49 patients. The lumbar CSF, pressure, and manometrics were examined and found normal in 46 cases. The Wassermann reaction was negative in the blood of 49 patients, and in the 46 specimens of CSF examined it was also normal. Appropriate percutaneous carotid angiography was carried out in 49 patients and, in all, the terminal portion of the common carotid and full extent of the internal carotid artery in the neck were visualized, as well as the intracranial circulation. In 45 patients the radiographs were normal; in four cases, two in each group, there was elongation of the internal carotid artery and a slight irregularity of the lumen, the appearances suggesting diffuse atheromatosis without stenosis.

\section{PROGRESS}

Figure 1 shows the follow-up in 25 patients who received only supportive treatment. Two patients died of unrelated cause: one, after $7 \frac{1}{2}$ years without neurological incident, of carcinoma of the bronchus, and the other, after $4 \frac{1}{2}$ years, of carcinoma of the rectum. One patient died, 14 months after presentation, with a verified myocardial infarction. One patient died of a stroke after three years. Another patient had a complete stroke after only three months. One patient has had multiple TIAs for $3 \frac{1}{2}$ years, but during the last 13 months has remained free of attacks, though he has minor hemiparesis. Another patient has had one TIA two years nine months after admission; he is well now, but has focal epilepsy. Two others have grand mal epilepsy, well controlled on treatment. One patient is troubled with the symptoms of vertebrobasilar insufficiency.

In summary, therefore, in the untreated group 


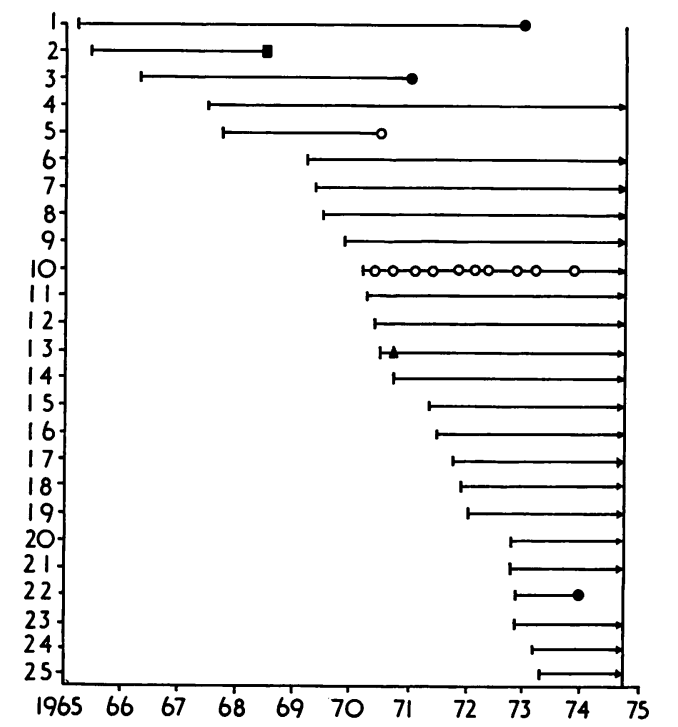

FIG. 1 No treatment group. $\square$ Died, stroke. Died, other cause. $\triangle$ Complete stroke. $\bigcirc$ Transient ischaemic attack or small stroke.

one patient has died of stroke and one has had a complete stroke. Two other patients have had transient strokes. Thus, from the neurological point of view, the result is satisfactory in 21 and fairly satisfactory in two cases; it was unsatisfactory in two cases.

Figure 2 shows the follow-up in 24 patients who have received anticoagulants: 22 received warfarin, two phenindione, for an average of 18 months (nine to 42 months). Six patients are still taking warfarin. One patient who suffered from gout developed a severe attack of gout three months after starting warfarin. It took many weeks to resolve and, because of wide fluctuations in prothrombin time, his warfarin was stopped. It was resumed nine months later after a small stroke which left him dysphasic; he has continued well to the present time. Three patients died of myocardial infarction: one three months after cessation of anticoagulant therapy, and another 20 months after; the third died of verified myocardial infarction while still on treatment. One patient had a TIA 19 months after finishing anticoagulants. Another patient had a small stroke 18 months after finishing warfarin, being left with dysphasia. Finally, one

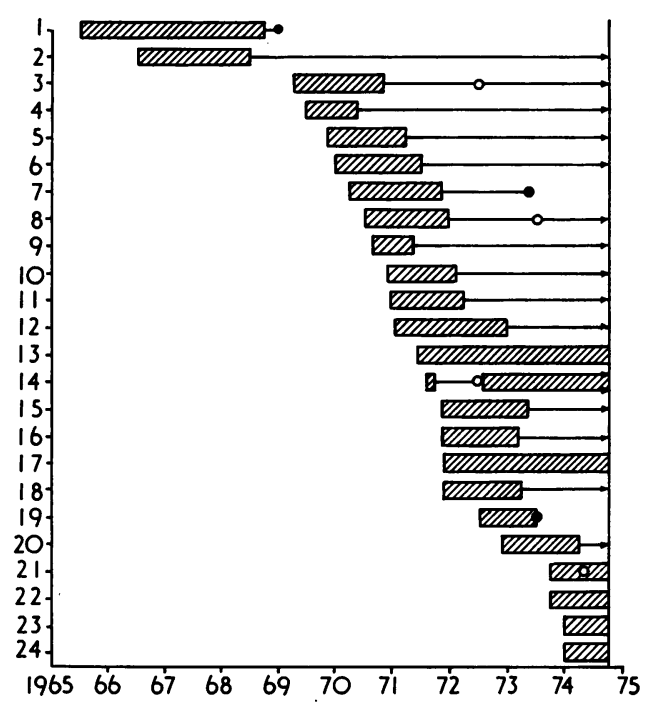

FIG. 2 Treatment group. Died, stroke. Died, $\stackrel{\infty}{\square}$ other cause. A Complete stroke. $\bigcirc$ Transientos ischaemic attack or small stroke. On anticoagulant.

patient had a TIA while on warfarin at months and remains well on warfarin still. Op patient has developed symptoms of vertebrobasilar insufficiency. No serious problems have arisen because of anticoagulant therapy.

In summary, in this group of 24 patients, none suffered severe strokes but two had small strokes $\varnothing$ while off treatment. However, one of these was in $\overrightarrow{\overrightarrow{0}}$ the patient with gout and we could not evaluate $\exists$ this properly because he had not had a full course of anticoagulants before they were stopped. A third patient had a TIA while off treatment and another had a TIA on treatment. Therefore, from the neurological point of view, the result is satisfactory in $\mathbf{2 0}$ patients and fairly $\delta$ satisfactory in three other cases. The fourth case $₹$ was unsatisfactory for follow-up but he remains $ᄋ$ stable after resumption of warfarin.

\section{DISCUSSION AND CONCLUSIONS}

The clinical features of 49 patients who sus- $\tilde{a}$ tained either a small sudden stroke or who had $N_{\omega}^{N}$ TIAs in the territory of the internal carotid artery have been presented. All were free from 0 relevant cardiac or other disease, were normo- 
tensive, and, in each, an appropriate percutaneous carotid arteriogram was normal. The patients were randomized into two groups: group group A comprising 25 who received only supportive treatment, and group B consisting of 24 who received anticoagulant therapy for an average period of 18 months. Analysis of age and sex distribution, duration of the neurological history, pattern of stroke presentation, the number of neurological episodes, and both the nature and extent of neurological signs showed no essential difference between the two groups. The average duration of the follow-up was 45 months in group A and 41 months in group B.

When the gross figures are broken down to the live patient months on and off treatment, the results were as follows. During the 1666 months off treatment, six patients had further neurological episodes which amounted to severe sudden stroke in two, mild sudden stroke in one, and TIAs in three others. One of those with multiple TIAs was found also to display minor pyramidal tract signs in hemiplegic distribution. One patient in this group was excluded for the purposes of analysis, and only progress after the recommencement of treatment, which had been interrupted on account of gout, has been considered. During the 448 live patient months on treatment, only one patient had a single TIA. Thus, there would seem to be some protection against further cerebrovascular accident during the time when anticoagulant therapy was administered. Comparison between the 543 live patient months after the cessation of anticoagulant treatment with the corresponding period in those who received only supportive treatment suggests that some marginal benefit may persist in the treated group, but the relatively benign nature of the syndrome and the small number of patients followed make any statistical analysis impossible.

Although no complications of anticoagulant treatment were encountered, this may be related to the personal supervision of patients by one interested consultant and, if such facilities are not available, it is likely that the disadvantages of anticoagulant treatment may outweigh the benefits.

It is a pleasure to record our thanks to Dr R. G. Grainger and Dr N. A. Lewtas for the radiological studies.

\section{REFERENCES}

Acheson, J., and Hutchinson, E. C. (1964). Observations on the natural history of transient cerebral ischaemia. Lancet, 2, 871-874.

Bradshaw, P., and Casey, E. (1967). Outcome of medically treated stroke associated with stenosis or occlusion of the internal carotid artery. British Medical Journal, 1, 201-205.

Bradshaw, P., and Gumpert, J. (1972). Small strokes in the carotid territory associated with a normal carotid arteriogram. Journal of Neurology, Neurosurgery, and Psychiatry, 35, 810-817.

Bradshaw, P., and McQuaid, P. (1963). The syndrome of vertebro-basilar insufficiency. Quarterly Journal of Medicine, 32, 279-296.

Fisher, C. M. (1958). The use of anticoagulants in cerebral thrombosis. Neurology (Minneap.), 8, 311-332.

Hill, A. B., Marshall, J., and Shaw, D. A. (1960). A controlled clinical trial of long-term anticoagulant therapy in cerebrovascular disease. Quarterly Journal of Medicine, 29, 597-609.

Hill, A. B., Marshall, J., and Shaw, D. A. (1962). Cerebrovascular disease: trial with long-term anticoagulant therapy. British Medical Journal, 2, 1003-1006.

Leishman, A. W. D. (1959). Hypertension-treated and untreated. A study of 400 cases. British Medical Journal, 1, 1361-1368.

Leishman, A. W. D. (1963). Merits of reducing high bloodpressure. Lancet, 1, 1284-1288.

McDevitt, E., Carter, S. A., Gatje, B. W., Foley, W. T., and Wright, I. S. (1958). Use of anticoagulants in treatment of cerebral vascular disease. Journal of the American Medical Association, 166, 592-597.

Marshall, J. (1963). Rebound phenomena after anticoagulant therapy in cerebrovascular disease. Circulation, 28, 329332.

Marshall, J. (1964). The natural history of transient ischaemic cerebro-vascular attacks. Quarterly Journal of Medicine, 33, 309-324.

Marshall, J. (1968). The Management of Cerebrovascular Disease. 2nd edn. Churchill: London.

Marshall, J., and Wilkinson, I. M. S. (1971). The prognosis of carotid transient ischaemic attacks in patients with normal angiograms. Brain, 94, 395-402.

Millikan, C. H., Siekert, R. G., and Shick, R. M. (1955). Use of anticoagulant drugs in the treatment of intermittent insufficiency of the internal carotid arterial system. Proceedings of the Staff Meetings of the Mayo Clinic, 30, 578586.

Millikan, C. H. (1971). Reassessment of anticoagulant therapy in various types of occlusive cerebrovascular diseases. Stroke, 2, 201-208.

Siekert, R. G., Millikan, C. H., and Whisnant, J. P. (1961). Anticoagulant therapy in intermittent cerebrovascular insufficiency. Journal of the American Medical Association, 176, 19-22. 